Presented at the Third National Conference

$L:--16458$

on Synchrotron Radiation Instrumentation at

Brookhaven National Laboratory, Sept. 12-14, 1983.

DESIS 006626

COMPUTED TOMOGRAPHY USING SYNCHROTRON RADIAT ION

H. C. Thompson and J. Llacer

Lawrence Berkeley Laboratory

University of California

Berkeley, CA 94720

L. Campbell Finman, E. B. Hughes, J.N. Ot is

S. Wj lson and H. D. Zeman

Hansen Laboratories of Physics

Stanford University

Stanford, CA 94305

September 1983

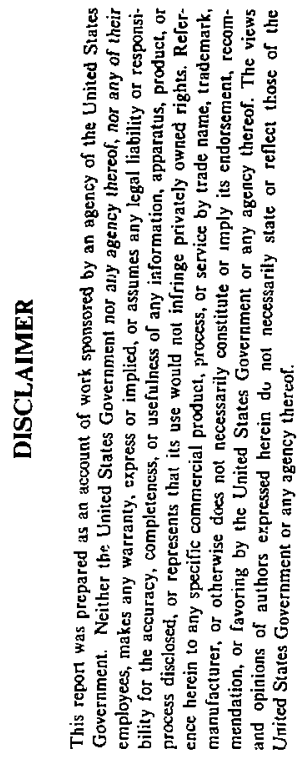

This work was supported in part by the Director's Office of Energy Research, Office of Health and Environmental Research, U.S. Department of Energy under Contract No. DE-ACO3-76SF00098, the Henry J. Kaiser Family Foundation and the National Science Foundation under Contract DMR 77-27489 (for the operation of SSRL). 


\title{
COMPUTED TOMOGRAPHY USING SYNCHROTRON RADIATION
}

\author{
A.C. Thompson and 1. Lacer \\ Lawrence Berkeley Laboratory \\ University of California \\ Berkeley, Ca, 94720.
}

L. Campbell Finman, E.B. Hughes, J.N. Ois,

S. Wilson and H.D. Zeman

Hansen Laboratories of Physics

Stanford University

Stanford, Ca. 94305 .

\begin{abstract}
$X$-ray computed tomography (CT) is a widely used method of obtaining cross-sectional views of objects. The high intensity. natural collimation, monochromaticity and energy runability of synchrotron $x$-ray sources could potentially be used to provide CT images of improved quality. The advantages of these systems would be that images could be produced more rapidly with better spatial resolution and reduced beam artifacts. In addition, images, in sone cases, could be acquired with elemental sensitivity. As a demonstration of the capability of such a system. CT images were obtainad of four slices of an excised pig heart in which the arteries and the cardiac chambers were filled with an iodinated medium. Images were taken with incidert $x$-rays tuned successively to energies just above and below the iodinc $K$ edge. Indine specific images were obtained by logarithmically subtracting the low energy image data from the high energy data and then reconstructing the image. CT imaging using synchrotron radiation may become a convenient and non-destructive method of imaging samples difficult to study by other methods.
\end{abstract}




\section{INTRODUCTION}

$\mathrm{X}$-ray computed tomography (CT) is a widely used method of obtaining cross-sectional views of obiects. This field was pioneered by Hounsfie]d [ 1$]$ in 1973 and now many commercial systems, using special high voltage x-ray tubes arc available, primarily for medical imaging. Synchrotron radiation sources potentially could provide a better source of $\mathrm{x}$-rays since they produce very intense, naturally collimated, narrow bandwidth and tunabic photon beams.

Synchrotron soutces offer several advantages for CT imaging. Principally, by taking two images above and below an $x$-ray zorpe en edge, it is possible to reconstruct the spatial distribution of a specific element. In addition, $t i c$ higt ur intensity could allow real-time imaging of moving samples, providing the samples could withstand rupid rotation. Beam hardening artifacts will not be present as the photons are almost monochromatic. I he background signal due to scattered photons is reduced since the beam has a divergence of only several mrad and the transmitted radiation can be collimated so that the detector sees very little scattered radiation. Radiation damage to the sample can be minimized by tuning the beam energy to give the maximum signal-to-noise ratio. A final advantage is that small samples could be studied with high spatial resolution in reasonable sampling times.

The application of synchrotron radiation to CT has teen studied theureticaliy by Grodzins [2] who found that water-like samples of $100 \mu \mathrm{m}^{2}$ size with a resolution element size of $1 \mu \mathrm{m}^{2}$ could be studjed whth a sensitivity below $50 \mathrm{ppm}$ for eiements from phosphorus to bromine. Many types of samples could be examined and elemental amages could be reconstructed providing the samples were thin enough to transmit enough $x$-rays near an appropriate absorption edge. Since the sample must be rotated. it may not be possible to use synchrotron sources for CT imaging of human subjects.

As a demonstration of the capability of a synchrotron CT system, trial images were obtained of four slices of an excised pig heart in which the arterial tree and the cardiac chambers were filled with iodinated vaseline. Images were taken with incident $x$-rays tuned successively to energies $j u s t$ above and below the iodine $\mathrm{K}$ edge. Iodine specific images were obtained by logarithmically subtracting the low energy data from the high energy data and then reconstructing the image.

\section{DATA ACQUISITION PROCEDURE}

The $x$-ray tomographic method requires the measurement of the $x$-ray transmission coefficients through each resolution element at a large number of angles. Usually the resolution element size is in the range 1 to $20 \mathrm{~mm}^{2}$ and more than 100 different angular measurernents are made. Using this data an image is then reconstructed of tue slice through the sample. There are a variety of algorithms which perform the reconstruction at high speed with modern minicomputers.

The method used in this study is shosn schematically in Fig. 1. The white radiation synchrotron beam is monochromatized, vertically collimated, passed through the sample and measured with a horizontal one-dimensional detector.

Specifically, a $2 \mathrm{~cm}$ wide beam from a wiggler insertion device at the Stanford Synchrotron Radiation Laboratory (SSRL) was monochromated by a standard SSRL double crystal monochromator to produce a beam with a fractional energy spread of about $5 \times 10^{4.4}$. The energy of the beam was selected by angular adjustment of the monochromator. The vertical height of the beam was collimated to $0.5 \mathrm{~mm}$ and the total beam intensity monitored with a standard SSRL argon-filled ionization chamber. The beam was then passed through the sample mounted on a computer controlled rotary stage. Following the sample was a one-dimensional multi-element detector which measured the beam intensity in horizontal segments of $0.5 \mathrm{~mm}$. This detector, which has been described previously by Thompson et a) [3], vias a cooled (-30 deg C) multi-element lithium-drifted silicon detector with a thickness of $5 \mathrm{~mm}$. The $x$-ray 
flux in each element was measured by conneciing a preamplifier and voltage-to-frequency converter :o each element, as described by Zeman et al [4], and measuring the frequency with a computer. This system has excellent dynamic range, linearity and stability. Since resolution elements of $0.5 \mathrm{~mm}$ by $0.5 \mathrm{~mm}$ were desired, but the center- to-center spacing of the detector elements was only $1 \mathrm{~mm}$, a comb sliz was placed ahead of the detectoi and two interlaced sub-images taken. In addition, since the beam width was only $2 \mathrm{~cm}$ and the feld-of-view necessary to cover the complete excised pig heart was $12 \mathrm{~cm}$, the sample was translated in six steps of $2 \mathrm{~cm}$ and the data acquired in swaths which were combined in correct sequence before the image was reconstructed.

A CT image was obtained by recording the intensity in each of the detector elements at different angular positions of the rotary sample stage. The intensities were normalized using the ionization chamber ahead of the sample. Elemenrally sensitive images were made by taking two sets of measurements, one just below an absorption edge of the element to be measured and the other just above the absorption edge. The elemental image was obtained by subtracting the normalized logarithmic intcnsity at each point. For indine-specific images one set of data was taken $25 \mathrm{eV}$ below the iodine $K$ edge at $33.169 \mathrm{keV}$ and another set $25 \mathrm{eV}$ above the edge.

The CT images were created using a Shepp-Logan[5] filter and back-projecting with linear interpolation. Synchrotron $x$-ray CT data has excellent statistics and difference images, in particular, have high contrast and sharp edges. Therefore, it is possible that the Shepp-Logan filter is not optimal and that a filter with a higher frequency cutoff would improve the reconstructed images.

\section{EXPERIMENTAL RESULTS}

The experiment was done during March, 1982 with the SSRL storage ring operating at $3 \mathrm{GeV}$ and $70 \mathrm{~mA}$. The beam intensity was about $4 \times 10^{9}$ photons $/ \mathrm{sec} / \mathrm{mm}^{2}$ at $33 \mathrm{keV}$. The detector and imaging system was identical to that used in an evaluation of the use of synchrotron radiation for non-invasive angiography. Results with this system have been presented by II\%ghes et al [6].

An embalmed pig heart was used as a sample for this study. Using appropriate mixtures of ethiodol (an iodine containing compound) and vaseline, the arterial tree, the left and right ventricles, and the right atrium were filled. The mixture was adjusted to make the iodine concentrations in the arterics $20 \mathrm{mg} / \mathrm{ml}$, in the left ventricle $20 \mathrm{mg} / \mathrm{ml}$, in the left atrium $13 \mathrm{mg} / \mathrm{ml}$ and in the right ventricle and right atrium $7 \mathrm{mg} / \mathrm{ml}$.

Two-dimensional $x$-ray pictures taken at incident photon energies $25 \mathrm{eV}$ above and below the jodine $\mathrm{K}$ edge at $33.17 \mathrm{keV}$ were first acquired at a variety of angles. The digitally subtracted logarithmic difference images wire then caiculated. A set of these images is shown in Figure 2. Fig. 2(a) is the low energy image, Fig 2(b) is the high energy image, Fig. 2(c) is the differcnce image and Fig. 2(d) is the difference image with the heart rotated 90 degrees. The four levels at which subsequent CT images were taken are marked by the numbered lines.

Normalized $x$-ray transmission data for the CT images were collected across a $12 \mathrm{~cm}$ width (240 resolution elements) and at angular steps of $1.8 \mathrm{deg}$ (100 projections). Four sets of data were acquired at different beights on the heart. The second set of reconstructed images taken above and below the jodine $\mathrm{K}$ edge at position 2 are given in Fig. 3(a-b). The four difference images are presented in Fig. 4 (a-d).

As can be seen very good images were oblained using synchrotron radiation. Although there are slight differences between the high and low energy images, the arteries are not visible in these images. However, when the difference images are calculated the arteries and ventricles stand out with excellent contrast. It is even possible to see that the larger arteries in these images are oblong since they are crossing the projection plane at an angle. 


\section{CONCLUSIONS}

CT images have been produced using a synchrotron $x$-ray beam line at SSRL. They demonstrate the ability to obtain both standard CT images and images which show the spatial distribution of only a specific element. When the capacity of tomographic imaging to produce cross-sectional images non-destructively is combined with a powerful synchrotron $x$-ray source a technique is provided to image samples which can not be studied with current $x$-ray sources. The high intensity, natural collimation, monochromaticity and tunability provide an almost ideal source for $x$-ray tomography. It can both improve CT imaging of samples by increasing the signal-io-noise ratio and also provide elemental sensitivity for many samples when the sample is thin enough to transmit $x$-rays near an appropriate absorption edge.

\section{ACKNOWLEDGEMENTS}

The assistance of $J$. Stone in preparation of the pig heart samples is apprcciated. The cooperation of the SSRL staff in the setup and operation of this experiment is gratefully acknowledged. This work was supported in part by the Director's Office of Energy Rescarch, Office of Health and Environmental Research, U.S. Department of Energy under Contract No. DE-AC03-76SF00093, the Henry J. Kaiser Family Foundation and the National Science Foundation under contract DMR 77-27489 (for the operation of SSRL).

\section{REFERENCES}

1. G.N. Hounsfield, Brit. J. Radiol. 46 (1973) 1016.

2. L Grodzins, Nucl. Instr. and Meth., 206 (1983) 541.

3. A.C. Thompson, F.S. Goulding, H.A. Sommer, J.T. Walton, E.B. Hughes, J. Rolfe and H.D. Zeman, IEEE Trans. Nucl. Sci., NS-29, (1982) 793.

4. H.D. Zeman, E.B. Hughes, L.E. Campbell, R. Hofstader, R.L Kirk. T.J. Krolicki, J. Rolfe, J.P. Stone, S. Wilson, E. Rubenstein, A.C. Thompson and J.T. Walton, IEEE Trans. Nucl. Sci., NS-29, (1982) 442.

5. L. A. Shepp and B.T. Logan, IEEE Trans. Nucl. Sci., NS-21, (1970) 21.

6. E.B. Hughes, H.D. Zeman, L.E. Campbell, R. Hofstader, U. Meyer-Berkout, J.N. Otis, J. Rolfe, S. Wilson, E. Rubenstein, D.C. Harrisol, R.S. Kernoff, A.C. Thompson and G.S. Brown, Nucl. Instr. and Meth., 208 (1983) 665. 


\section{FIGURE CAPTIONS}

1. Schematic layout of a computed tomographic imaging system using synchrotron radiation.

2. Two-dimensional $x$-ray images of an embalmed pig heart. Fig. 2(a) is an image taken at 25 eV below the iodine $\mathrm{K}$ edge, Fig. 2(b) is the same image taken at $25 \mathrm{eV}$ above the cdge and Fig $2(\mathrm{c})$ is the difference image taken at the same position. Fig. $2(d)$ is the difference image taken with the pig heart rotated 90 degrees to Fig. 2(c). The four positions at which CT images were obtained is indicatcd by the nunbers $1-4$.

3. CT images obtained at incident photon energies $25 \mathrm{eV}$ below , d) and above (b) the iodine $\mathrm{K}$ edge at vertical position 2 of the heart.

4. CT difference images obtained by logarithmically subtracting the low energy image data from the high energy smage data before image reconstruction. The four images correspond to the four positions indicated in Fig 2. 


\section{COMPUTED TOMOGRAPHY USING SYNCHROTRON RADIATION}

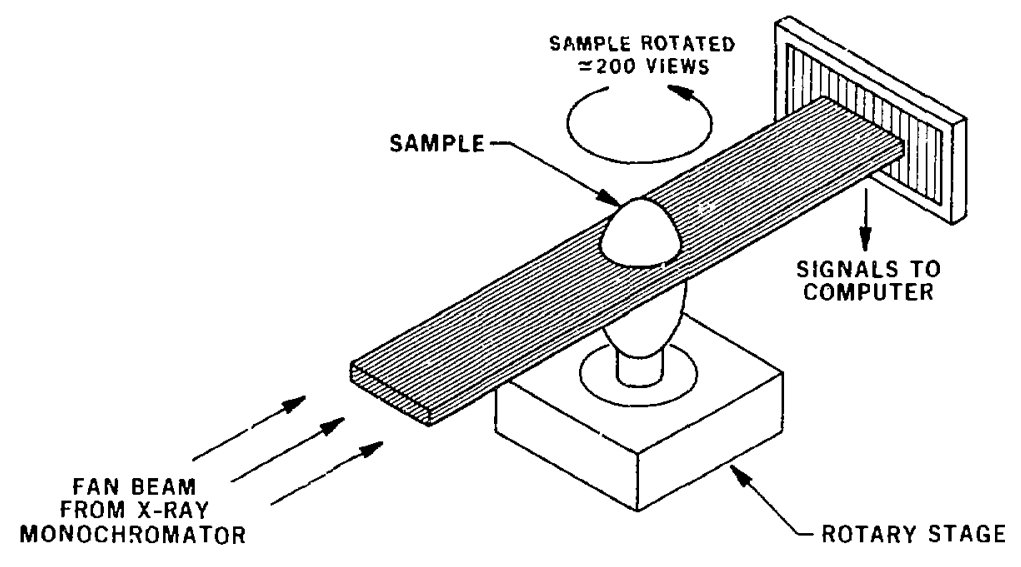

XBL $8210-2908$

Fig. 1. Schematic layout of a computed tomographic imaging system using synchrotron radiation. 


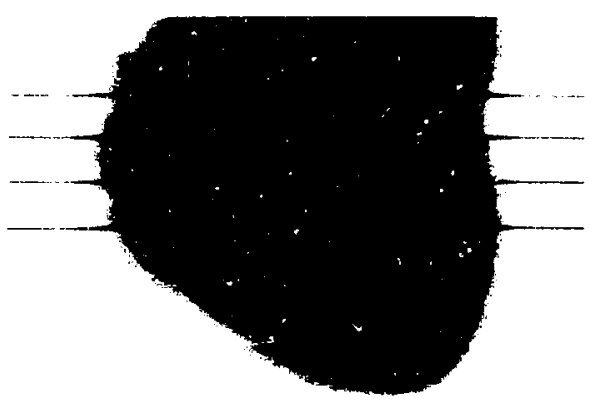

a)

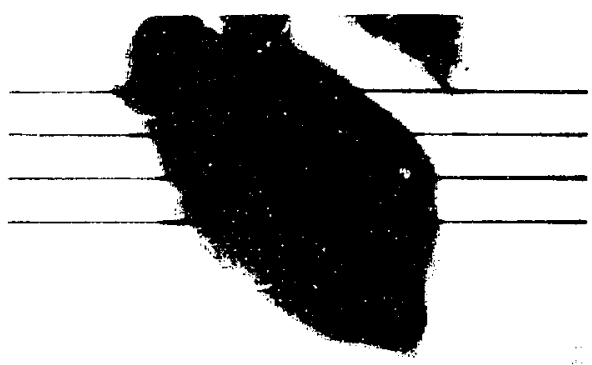

c)

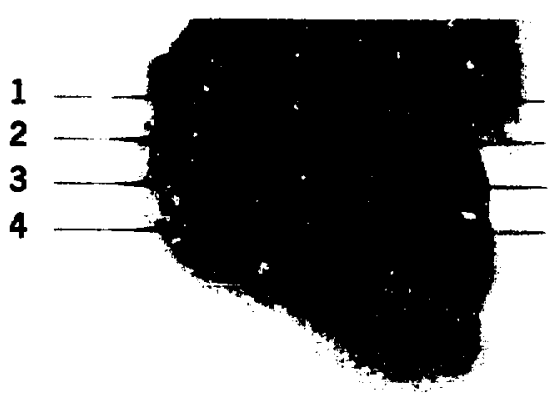

b)

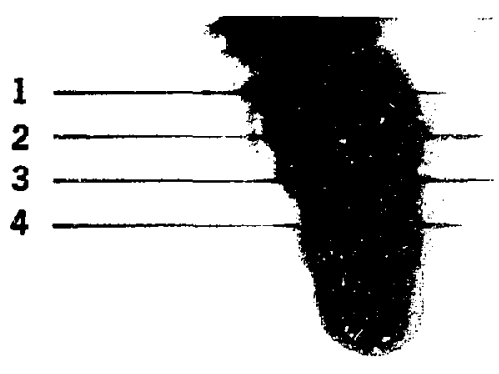

d)

$\times 3 B \quad 830-9220$

rig. 2. Two-dmensional $x$-ray images of an embalmed pig heart. Tigurt: $2(a)$ is an image taken at $25 \mathrm{eV}$ below the iodine $K$ edge, Fig。 $2(b)$ is the sar:e inar. taken at $25 \mathrm{eV}$ above the edge and Fig, $2(\mathrm{c})$ is the difference inage lat an at the same position. Figure $2(d)$ is the difference illare taken with the win meart rotated 90 degrees to $\mathrm{Fig}_{0} 2(\mathrm{c})$. The four positions at which r I illuses were obtained is indicated by the numbers $1-4$. 


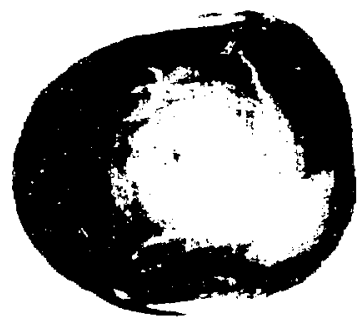

a)

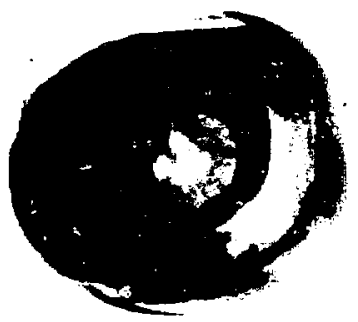

b)

XBB $830-9218$

Fig, 3. CT images obtained at incident photon energies 25 eV beluw (a) and above (b) the iodine $K$ edge at vertical position 2 t the heart. 


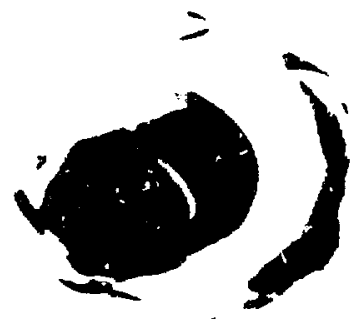

a)

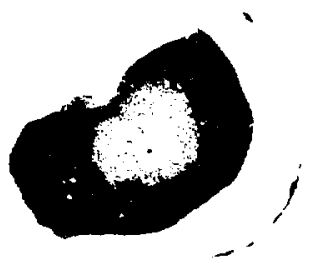

c)

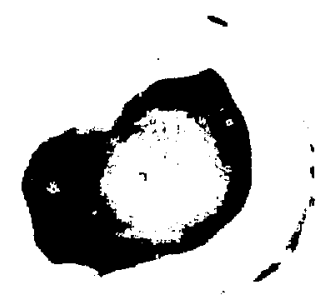

b)

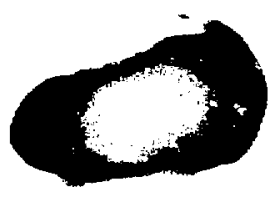

d)

$X B B \quad 830-0: 2 !:=$

Fig. 4. CT difference images obtained by logarithmically subtracting the low energy image data from the high energy image data before image reconstruction. The four images correspond to the four positions indicated in Fig. 2. 
This report wis done with support from the Departenent of Energy. Any conclusions or opinions expressed an this report icpresent solely those of the author(s) and not necessarily those of The Regents of the University of Californta, the l.aurence Berkeley l.aboratory of the Department of Energy.

Reference lo a company or producl name does not imply approval of ecomnendation of the product by the University of Californin ar the U.S. Department of Energy to the exclusion of athers that may be suitable 